\title{
Effect of the microstructure closed pore content on the acoustic behavior of polyurethane foams
}

\author{
Olivier Doutres, ${ }^{1, a)}$ Noureddine Atalla, ${ }^{1}$ and Kevin Dong ${ }^{2}$ \\ ${ }^{1}$ GAUS, Department of Mechanical Engineering, Université de Sherbrooke, Quebec JIK $2 R 1$, Canada \\ ${ }^{2}$ Woodbridge Foam Corporation, Chemical Development Center, 8214 Kipling Avenue, Woodbridge, \\ Ontario L4L 2A4, Canada
}

(Received 5 May 2011; accepted 21 July 2011; published online 19 September 2011)

\begin{abstract}
The present paper proposes to investigate the links between the microstructure of polyurethane foams and their sound absorbing efficiency, and more specifically the effect of membranes closing the cells. This study is based on the complete characterization of 15 polyurethane foam with various cell sizes and reticulation rates (i.e., open pore content): (i) characterization of the microstructure properties (cell size $C_{s}$, strut thickness $t$, reticulation rate $R_{w} \ldots$ ) from SEM pictures, (ii) characterization of nonacoustic parameters (porosity $\Phi$, airflow resistivity $\sigma$, tortuosity $\alpha_{\infty} \ldots$ ) from direct and indirect methods. Existing analytical links between microstructure properties and nonacoustic parameters are first applied to fully reticulated materials. Then, they are improved empirically to account for the presence of the closed pore content. The proposed expressions associated to the Johnson-Champoux-Allard porous model allow a good estimation of the sound absorbing behavior of all polyurethane foams, fully reticulated or not. This paper also demonstrates the important effect of the presence of cell membranes: increase of the airflow resistivity, tortuosity, and the ratio between the thermal and viscous characteristic lengths while decreasing these two characteristic lengths. Thus, the sound absorption efficiency at low frequencies is improved but can be worsened in some higher frequency bands. (C) 2011 American Institute of Physics. [doi:10.1063/1.3631021]
\end{abstract}

\section{INTRODUCTION}

Since long time, chemists know how to control the foaming process of polyurethane foams to access the desired microstructure. Especially, the choice of raw materials (i.e., polyol, di-isocyanine, water, auxiliary blowing agents, catalysts, surfactant and other additives) allows to control the gas evolution and the polymerization of the cell wall and struts during foaming, resulting in targeted foam density, mechanical strength, cell size and cell opening. At the same time, the scientific community specialized in porous media developed material models to account for the acoustic energy dissipation associated to the propagation of an acoustic wave inside the porous microstructure. ${ }^{1}$ The models are mainly semiphenomenological because a complete analytical description of sound propagation in complex porous microstructure in not possible. Thus, several physical parameters are involved to describe the viscothermal and inertial couplings between the porous aggregate and the interstitial fluid on a macroscopic scale: e.g., open porosity $\Phi$, static air flow resistivity $\sigma\left[\mathrm{N} \mathrm{s} / \mathrm{m}^{4}\right]$, dynamic tortuosity $\alpha_{\infty}$, viscous $\Lambda[\mu \mathrm{m}]$, and thermal characteristic lengths $\Lambda^{\prime}[\mu \mathrm{m}]$ and thermal static permeability $k_{0}{ }^{\prime}\left[\mathrm{m}^{2}\right]$. However, the interpretation of these parameters, here referred to as the nonacoustic parameters, in terms of foam microstructure remains unclear and has to be improved since it is the key to an optimization of the foaming process.

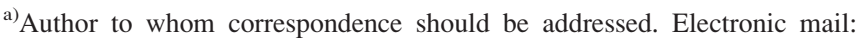
olivier.doutres@usherbrooke.ca.
}

Different approaches have been proposed in the literature to improve the interpretation between microstructure and nonacoustic parameters. First, empirical methods have been developed from the analysis of the exact nature of the microstructure and the measurement of the acoustic performance. $^{2-6}$ These models, mainly developed for fibers, give an estimation of the airflow resistivity $\sigma$ from the bulk density of the fibrous material $\rho_{1}$ and the fiber diameter $d$. It is found that the relation between these three parameters depends on the type of material (glass wool, polyester fibers...) under investigation; which is characteristic of empirical approaches, but can nevertheless be used to optimize the fabrication process of the considered porous material.

A second approach is based on scaling laws that link macroscopic behavior to microstructure from simplified models of the microstructure ${ }^{7,8}$ (cell shape) and wave propagation inside this microstructure. ${ }^{1,9,10}$ For example, Allard and Atalla ${ }^{1}$ give an analytical expression linking the viscous characteristic length $\Lambda$ and the fiber diameter of fibrous materials from the case where the velocity of the air is perpendicular to the direction of the fibers. From geometrical considerations of an idealized cell which constitute the porous material, Gibson and Ashby ${ }^{7}$ give simple analytical expression linking the porosity $\Phi$ and microstructure properties such as strut length $l$ and thickness $t$. Recently, Perrot et al. ${ }^{8}$ adapted this expression for open-cell aluminum foams considering an idealized unit-cell with tetrakaidecahedra shape, and in the same way, proposed an expression for the thermal characteristic length $\Lambda^{\prime}$. Finally, by combining some of the expressions previously mentioned, Lind-Nordgren and Göransson $^{9,10}$ proposed a scaling law applied to the airflow 
resistivity $\sigma$ of porous materials which links this nonacoustic property to cell and strut shapes.

A final approach uses numerical methods. ${ }^{811-14}$ The idealized periodic unit cell is then reconstructed, using $\mathrm{x}$-ray tomography $(\mu$-san) and advanced computational fluid dynamics analysis is performed to derive properties relevant for the acoustic behavior. These methods allow a great understanding of the impact of microstructure on acoustic behavior. However they are experimentally limited to specific materials (visible with x-ray tomography) and are computationally intensive; they are still not able to represent realistic microstructures such as those found in partially reticulated foams.

In this paper, the micro-macro links and more especially the effect of membranes closing the cells are investigated using a semiempirical approach based on 15 polyurethane foams. These foams, whose microstructure is show in Fig. 1, mainly vary by their cell size and closed cell content. According to this figure, the polyurethane foam microstructure can be seen as a collection of interlinked struts forming 3D structures as a packing of tetrakaidecahedra cells. Each cell is connected to other through pores. Materials with open pores are called "fully reticulated." In this case the interconnectivity between cells is maximum. If some of the pores are closed or partially closed by thin membranes, the material is called "partially reticulated." The approach proposed in this paper is to (i) analyze the exact nature of the 15 polyurethane foams microstructure using a scanning electron microscope (SEM), (ii) measure their nonacoustic properties, (iii) validate and apply the existing scaling law to the fully reticu-

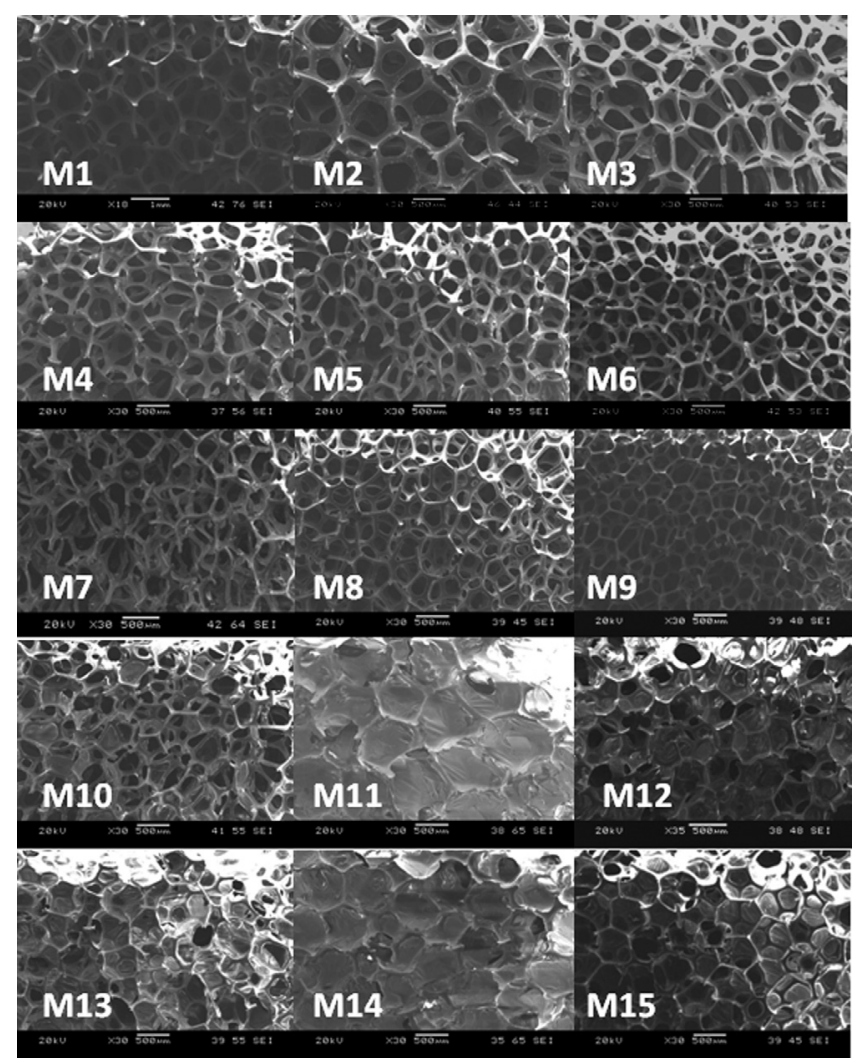

FIG. 1. SEM pictures of the 15 polyurethane foam materials. lated polyurethane foams, and (iv) improve them to account for the presence of membranes closing the pores using geometrical or empirical analysis. An emphasis is thus proposed on the influence of the reticulation rate of the porous microstructure. Despite the fact that empirical expressions are not general and cannot be applied to various porous microstructures, this study presents tools to improve the understanding on the consequence of foam microstructure and reticulation rate on acoustical behavior.

\section{FOAM CHARACTERIZATION}

\section{A. Microstructure}

Microstructure of the 15 polyurethane foams, called M1 to M15, is analyzed from SEM pictures taken at University of Toronto and presented in Fig. 1. One can easily appreciate the different foam microstructure since SEM pictures with identical properties are presented here (the magnification is set to $\times 30$ except for materials with reference M12 with $\times 35$ and M1 with $\times 18$ ). Thus, it is shown that the materials mainly differ by their cell size and the presence or lack of membranes closing the faces of the cells. As Perrot et al. ${ }^{8}$ did in the case of open-cell aluminum foams, the microstructure of polyurethane foam is seen as a packing of tetrakaidecahedra cells (see Fig. 2 taken from Ref. 8) characterized by $f=14$ faces/cell and $n=5.14$ edges/face and independent from cell density. The main objective here is to determine, for each material, the properties of a representative tetrakaidecahedra unit-cell (also called PUC in Ref. 8) from which the existing scaling laws would be applied.

As shown in Fig. 2, the size of the unit-cell $C_{s}[\mu \mathrm{m}]$ is an important parameter. Because of the fabrication process, the foams may have elongated cells resulting in cell size along three dimensions $\left(C_{s 1}=2 R_{1}, C_{s 2}=2 R_{2}, C_{s 3}=2 R_{3}\right)$. The degree of anisotropy DA given by $\mathrm{DA}=C_{s 3} / C_{s 1}=$ $R_{3} / R_{1}$ is measured for the 17 polyurethane foams initially under investigation. The anisotropy threshold is set here to $\mathrm{DA}=1.25$ and it is found that 15 materials on 17 can be considered as isotropic $\left(C_{s}=C_{s 1}=C_{s 2}=C_{s 3}\right)$. In this work, since

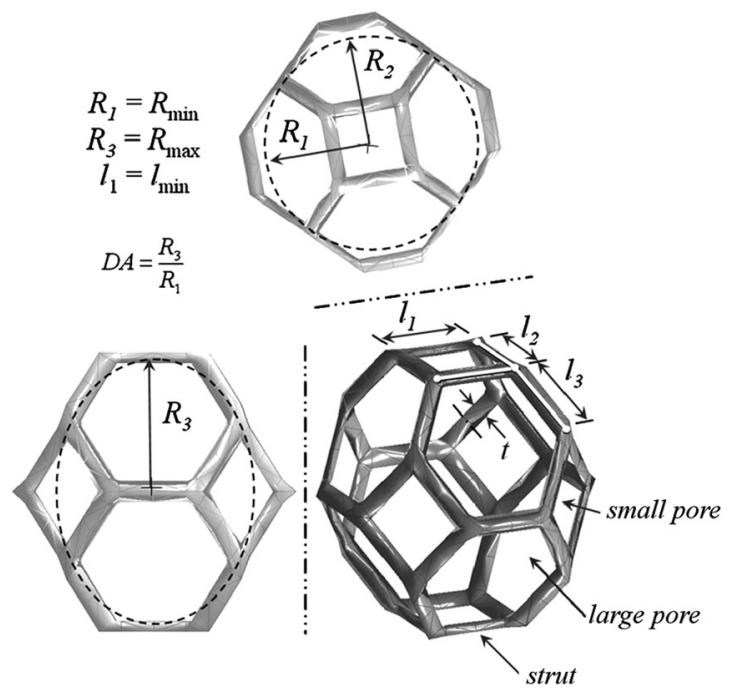

FIG. 2. The shape and size parameters measured on a unit cell (taken from Ref. 8). 
we are interested in linking cell microstructure to nonacoustic parameters from simple models, only the 15 materials having isotropic cells are considered in the analysis. For each material, mean strut length $l[\mu \mathrm{m}]$ and thickness $t[\mu \mathrm{m}]$ are also measured. Strut length $l$ is derived as the mean value between the three lengths $l_{1}, l_{2}$, and $l_{3}$ as show in Fig. 2 and detailed in Ref. 8 The struts of polyurethane foams having triangular cross-section shape (see Fig. 1), only the edge $a$ of the triangle is measured on SEM pictures. The strut thickness $t$ is the height of this triangle assumed equilateral and thus given by $t=a \sqrt{3} / 2$. In order to complete the cell description, the small pore size $W_{s}[\mu \mathrm{m}]$, large pore size $W_{l}[\mu \mathrm{m}]$ and the mean value $W_{m}$ are also measured (see Fig. 2). Finally, the reticulation rate $R_{w}$ [\%], which gives the open window content of the material, is estimated from SEM pictures by the ratio of the number of open window to the total number of windows visible on the pictures. Nine of 15 materials are fully reticulated $\left(R_{w}=100 \%\right)$ and the six partially reticulated materials have a reticulation rate ranging from $10 \%$ to $70 \%$. It is worth noting that since very low reticulation rate have been measured, it is assumed that the cutting has no influence on the absence of cell membranes.

For each material, the unit-cell properties described previously are measured on different SEM pictures taken at various locations and using the "Image J" software (http:// rsbweb.nih.gov/ij/). In order to ensure that the characterized unit-cell is representative of a mean cell for each material, all properties and associated expanded uncertainties ${ }^{15}$ are determined from a large number of measurements: e.g., 10 measurements for $C_{s}, 20$ for $t, l$, and $W_{m}$ and between 100 and 200 windows are counted to estimate $R_{w}$. Each polyurethane foam is now characterized by an idealized periodic cell (PUC) with the associated uncertainty on each property; which ensures its representativeness.

Figure 3 presents microstructure properties evolution in terms of cell size. Here, the 15 isotropic polyurethane foams have cell sizes ranging from 500 to $1600 \mu \mathrm{m}$. It is shown that the strut thickness $t$, strut length $l$ and window size $W_{m}$ increase in a linear way with the increasing cell size for both fully reticulated $\left(R_{w}=100 \%\right)$ and partially reticulated materials $\left(R_{w}<100 \%\right)$. Also, it is found that the strut length to thickness ratio $l / t$ and the cell size to window size ratio $C_{s} / W_{m}$ are almost identical for all cell size. This indicates that from one cell size to another, the shape of the cells is unchanged and their size parameters are only magnified by a given factor. It is worth noting that, as expected, the cell size is ranging between two and three times the window size; its average is around $C_{s} / W_{m}=2.88$. All these observations corroborate what have already found by Perrot et al. ${ }^{8}$ in the case of metallic aluminum foams.

\section{B. Nonacoustic properties}

This section presents the nonacoustic properties of the 15 polyurethane foams (e.g., open porosity $\Phi$, static air flow resistivity $\sigma\left[\mathrm{N} \mathrm{s} / \mathrm{m}^{4}\right]$, dynamic tortuosity $\alpha_{\infty}$, viscous $\Lambda$ $[\mu \mathrm{m}]$, and thermal characteristic lengths $\Lambda^{\prime}[\mu \mathrm{m}]$ and thermal static permeability $\left.k_{0}^{\prime}\left[\mathrm{m}^{2}\right]\right)$. In this paper, an equivalent fluid model $^{1}$ is used to describe the propagation of an acoustic wave in a rigid frame porous. This model assumes that the frame is motionless and is not subjected to deformation. The dynamic heat conduction phenomena in the open-cell porous media is described using the Champoux-Allard model modified by Lafarge et al. ${ }^{16}$ and the viscous dissipation from the Johnson et al. model. ${ }^{17}$ Note that these nonacoustic parameters are intercorrelated and morphology-dependent.

As mentioned previously, porous bulk density $\rho_{1}$ $\left[\mathrm{kg} / \mathrm{m}^{3}\right]$ is also measured because it is an important data. Indeed, the frame density generally guides the possible foam application (e.g., light foams for cars or planes, heavy foams for houses...). This parameter is also required in two porous models which account for the elasticity and inertia of the frame, i.e., the Biot-Allard model and the "limp frame" equivalent fluid model. ${ }^{1}$ Here, the inertia of the frame is not taken into account since the considered "rigid frame" equivalent fluid model used in this study considers that the frame is rigid and motionless. Other properties exist but are not investigated in this report since the main six properties are usually sufficient for engineering analysis in the context of building or transport applications. Since foams with anisotropic cell shape have been discarded from the present analysis, the foams are assumed isotropic at the macroscopic level.

The bulk density $\rho_{l}$, the porosity $\Phi$ and the airflow resistivity $\sigma$ are determined from direct measurements. ${ }^{18,19}$ Airflow resistivity of each sample is measured for various static airflow (between $1.58 \times 10^{-6} \mathrm{~m}^{3} / \mathrm{s}$ and $2.75 \times 10^{-6}$ $\mathrm{m}^{3} / \mathrm{s}$ ) and its real value is considered as the asymptotic value for a airflow equal to $0 \mathrm{~m}^{3} / \mathrm{s}$. For both fully and partially


FIG. 3. Microstructure properties of the 15 polyurethane foams. Expanded uncertainty bars are also shown. 
reticulated foams, the tortuosity $\alpha_{\infty}$ is estimated from direct method based on ultrasound technique. Tortuosity of fully reticulated materials is determined by acoustical techniques as an ultrasonic measurement of transmitted waves. ${ }^{20}$ This latter method being restricted to low resistive materials, the tortuosity $\alpha_{\infty}$ of partially reticulated foams is estimated from the measurement of acoustic waves reflected by a slab of porous material at oblique incidence. ${ }^{21}$

The two characteristic lengths $\left(\Lambda, \Lambda^{\prime}\right)$ and the thermal permeability $k_{0}^{\prime}$ are determined using the indirect characterization method proposed by Panneton and Olny. ${ }^{22,23}$ This requires the measurement of the equivalent dynamic bulk modulus $K_{\mathrm{eq}}=K / \Phi$ and equivalent dynamic density $\rho_{\mathrm{eq}}$ $=\rho / \Phi$ of the tested material performed here using the 3-microphone impedance tube method proposed by Salissou and Panneton. ${ }^{24}$ The indirect method associated to the 3-microphone impedance tube method has been recently detailed by the authors in Ref. 25 . The tube used for the measurements has a $44.5 \mathrm{~mm}$ inner diameter and the loudspeaker at one end generates a broadband random signal in the frequency band $200-4100 \mathrm{~Hz}$. The determination of the three parameters $\left(\Lambda, \Lambda^{\prime}, k_{0}^{\prime}\right)$ is considered satisfactory when both the measured equivalent dynamic bulk modulus and equivalent dynamic density of the materials are correctly predicted as shown in Fig. 4 for material M5 (in this figure, equivalent dynamic bulk modulus and equivalent dynamic density are normalized by the atmospheric pressure $P_{0}$ and the density of air $\rho_{0}$, respectively). Note that for partially reticulated materials, i.e., materials with higher airflow resistivity, the material was loaded with nails to suppress or minimize the frame vibrations and in consequence the effect of mounting conditions. This is of great importance since the characterization method assumes a rigid-frame behavior. Despite this precaution, the influence of mechanical resonances was too important above $2.5 \mathrm{kHz}$; which explains the frequency limitation presented in Fig. 4.

The Lafarge et al. model is used here to model the equivalent dynamic bulk modulus $K_{\text {eq }}$ because it was shown elsewhere to match more exactly its low frequency behavior. ${ }^{1}$ This implies the determination of the parameter $k_{0}^{\prime}$ but is aimed here to lower the error in the determination of the thermal characteristic length $\Lambda^{\prime}$. Indeed, since the thermal permeability parameter is not directly linked to microstruc- ture geometry element or to another nonacoustic parameter, it will be discarded from the micro/macro analysis, at least for the time being.

Finally, as mentioned by Olny and Panneton, ${ }^{23}$ the parameters measured with the indirect method cannot be considered as exact values for the true parameters since they depend on the initial model, which is not exact itself. The soobtained solutions are only the best fitted parameters for the model under consideration. It should be mentioned that both the Johnson et al. and Lafarge et al. model were improved $^{26,27}$ in order to match more exactly the frequency behavior of $K_{\text {eq }}$ and $\rho_{\text {eq }}$ introducing more intrinsic geometrical parameters. However, due to the actual difficulties to measure or determine these parameters, they are not studied in this paper.

Note that the nonacoustic properties of each foam have been determined from measurements carried out on three different samples and tested with the same mounting conditions. Thus, all values are averages given with their associated expanded uncertainties. In the specific case of the "pressure/mass" method to determine both the open porosity and the bulk density, a large bulk volume of material has been used to reach a good precision of measurement. ${ }^{18}$

\section{Fully reticulated materials}

Figure 5 presents the measurements of the nonacoustic properties of the nine fully reticulated materials in terms of cell size (the closed pore content per cell is equal to $0 \%)$.

The porosity is not influenced by cell size as shown in Fig. 5(a). Figure 5(b) confirms that the airflow resistivity $\sigma$ decreases with increasing the cell size. ${ }^{13,14}$ For cell size ranging from 500 to $1500 \mu \mathrm{m}$, the airflow resistivity varies from 600 to $4000 \mathrm{~N} \mathrm{~s} \mathrm{~m}^{-4}$ which is considered as a relatively low airflow resistivity for typical sound absorbing materials. Figure 5(c) shows that, the tortuosity $\alpha_{\infty}$ of fully reticulated materials is close to 1 and that this property does not vary with cell size. Finally, it is observed in Figs. 5(d) and 5(e) that both viscous and thermal characteristic lengths increase with cell size. The viscous characteristic length $\Lambda$ is found approximately 0.35 times the cell size $\left(\Lambda=0.35 C_{s}\right)$, making it comparable to the mean pore size (i.e., for fully reticulated
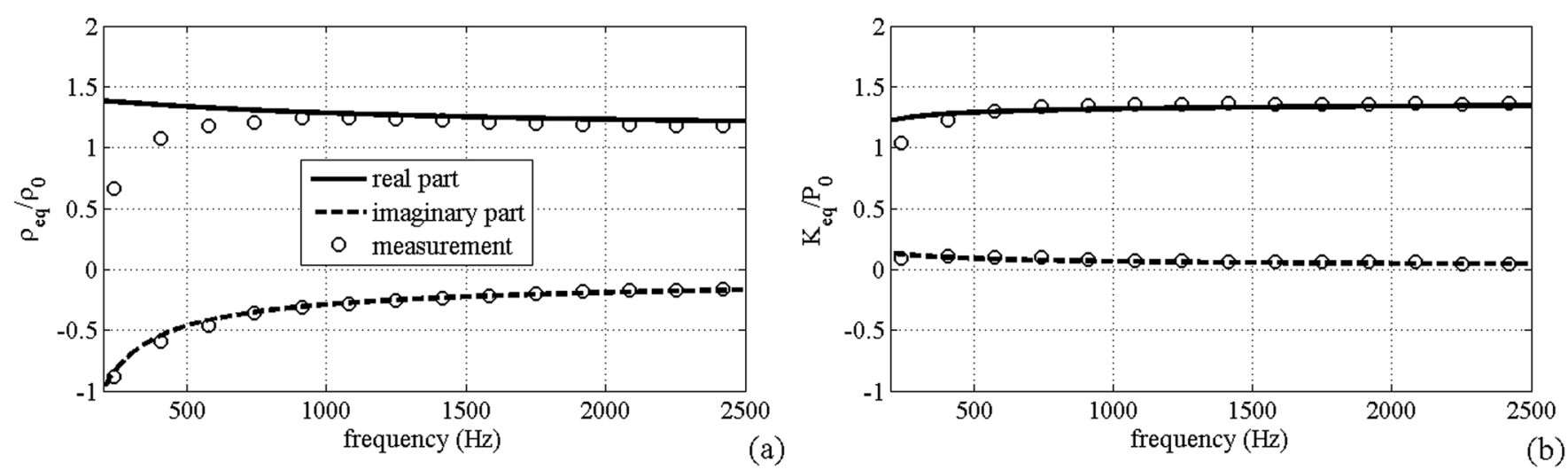

FIG. 4. Measurement and simulation of the normalized equivalent density and normalized equivalent bulk modulus of material M5. 


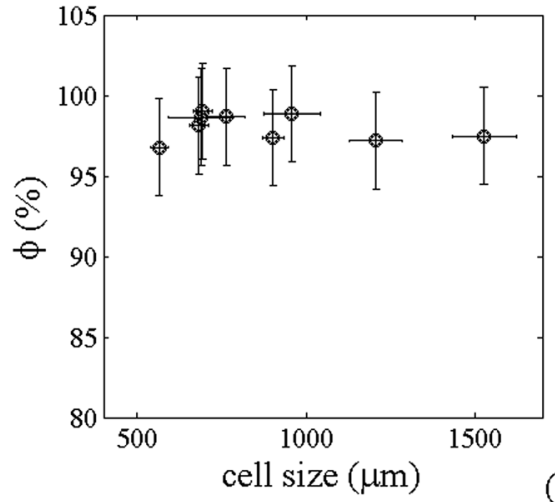

(a)

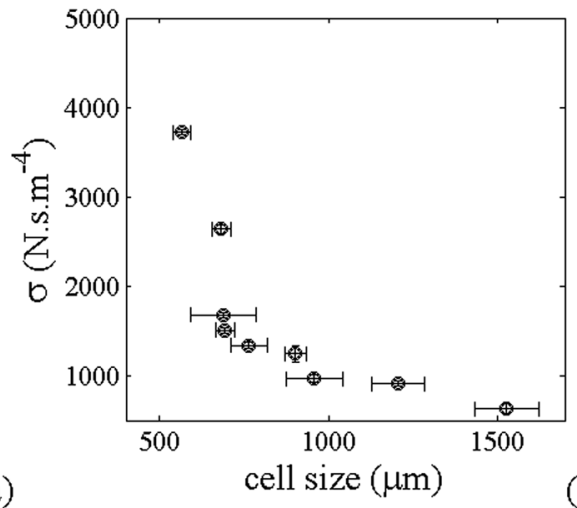

(b)

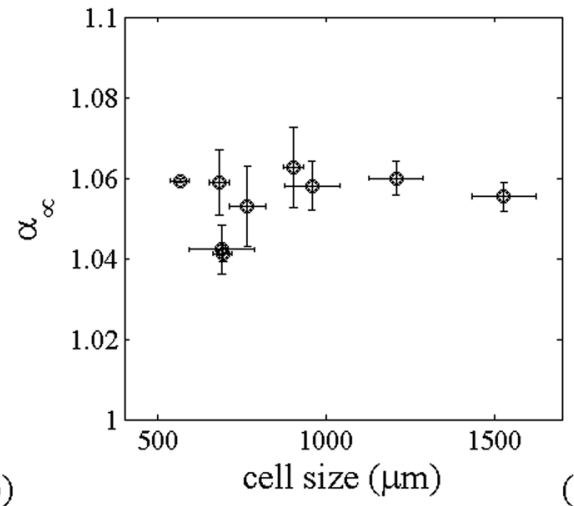

(c)
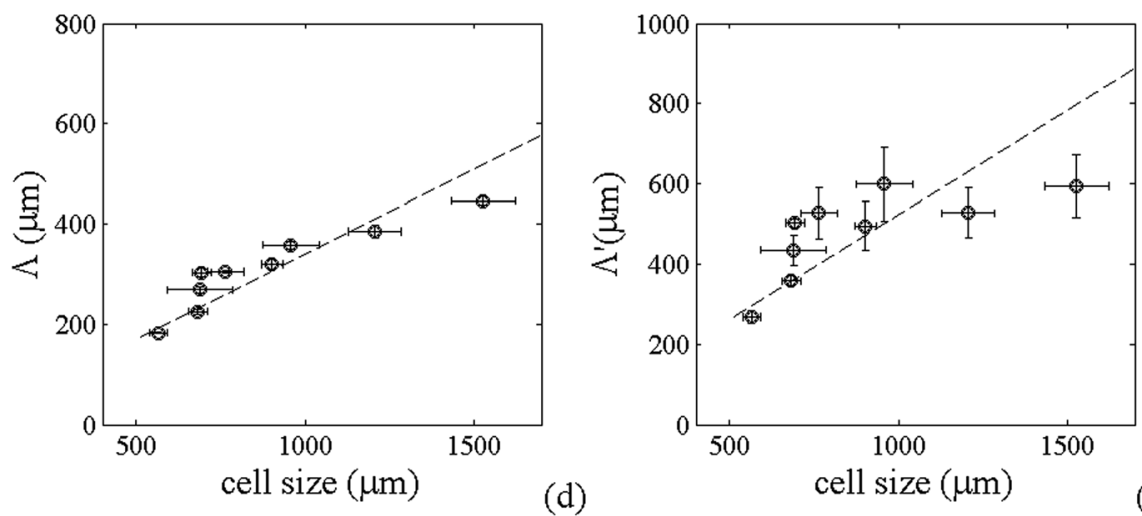

(d)

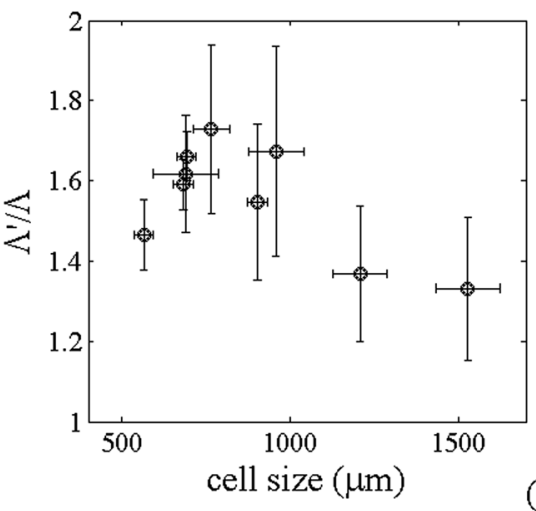

(f)

FIG. 5. Measured nonacoustic parameters of the 9 fully reticulated polyurethane foams in terms of cell size.

materials $W_{m}=0.34 C_{s}$ ). Furthermore, thermal characteristic length $\Lambda^{\prime}$ is found to be 0.56 times the cell size $\left(\Lambda^{\prime}=0.56 C_{s}\right)$. The ratio of these two characteristic lengths, $n=\Lambda^{\prime} / \Lambda$, seems to decrease with the increasing cell size. However due to its large associated standard deviation, it is considered that it does not depend on cell size and is equal to the mean value $n=1.55 \pm 0.2$, which is close to 2 and agrees with the value given in Ref. 1 (see details in Sec. III A 2 of the present paper).

\section{Partially reticulated materials}

The six partially reticulated materials considered in this study have a closed pore content $\left(1-R_{w}\right)$ ranging from $30 \%$ to $90 \%$. Figure 6 presents the measurements of the nonacoustic properties of these materials in terms of the closed pore rate.

The porosity is not influenced by the reticulation rate as shown in Fig. 6(a). The cells are thus highly interconnected even for low reticulated materials. In terms of modeling, high porosity is consistent even for low reticulated materials, e.g., $1-R_{w}=90 \%$, since the material microstructure is regarded here as a packing of interconnected cells through the reticulation rate shared between the cell pores, and not as a randomly packing of $90 \%$ closed cell and $10 \%$ open cell which would have created a much lower porosity material. Figures 6(b) and 6(c) show that both the airflow resistivity $\sigma$ and the tortuosity $\alpha_{\infty}$ increase with the closed pore content; which is in good agreement with numerical predictions of Boeckx et al. ${ }^{14} \mathrm{~A}$ maximum airflow resistivity is found at $41600 \mathrm{~N} \mathrm{~s} \mathrm{~m}^{-4}$ and the tortuosity at 2.4.
For these partially reticulated materials, the influence of cell size $C_{s}$ on nonacoustic properties can decrease at the expense of the closed pore rate $\left(1-R_{w}\right)$. The influence of these two microstructure properties on nonacoustic parameters is presented in Fig. 7. For example, in the case of the tortuosity $\alpha_{\infty}$ [see Figs. 6(c) and 7(c)], the closed pore content becomes the only influent parameter; i.e., the material with the higher closed pore rate has the higher tortuosity. However, in the case of the airflow resistivity $\sigma$, the cell size $C_{S}$ still has an important influence. Thus, as shown in Figs. 6(b) and 7(a), the material with an intermediate closed pore content of $73 \%$ shows the higher airflow resistivity because it has the smallest cell size.

Figure 6(d) shows that the viscous characteristic length $\Lambda$ decreases with the close pore content. This trend is not so clear in the case of the thermal characteristic length $\Lambda^{\prime}$ [see Fig. 6(e)]. Thus, the effect of the presence of membranes in the microstructure is also presented. This influence is derived from the difference between the estimated characteristic length if the material had no membranes (e.g., $\Lambda_{\text {th }}^{\prime}=0.56 C_{s}$ obtained for fully reticulated materials, see Sec. II B 1) and the measured characteristic length (e.g., $\Lambda_{\mathrm{m}}^{\prime}$ ). Results presented with white squares in Figs. 6(d) and 6(e) show that the influence of the reticulation rate increases as the closed pore content increases. This behavior corroborates the theoretical definitions of $\Lambda^{\prime}$ and $\Lambda$. Indeed, for a given cell size, i.e., cell volume, the two characteristic lengths decrease with the presence of membranes since it increases the wet surface and the local fluid velocity in the cells.

As for the airflow resistivity, both viscous and thermal lengths of partially reticulated materials still depend on cell 

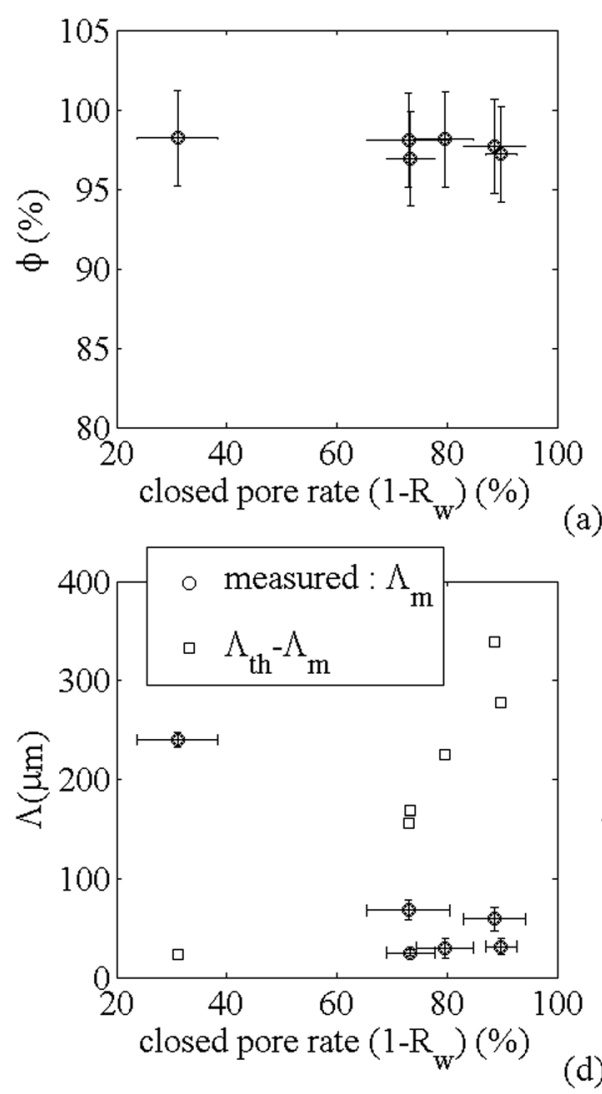

(a)
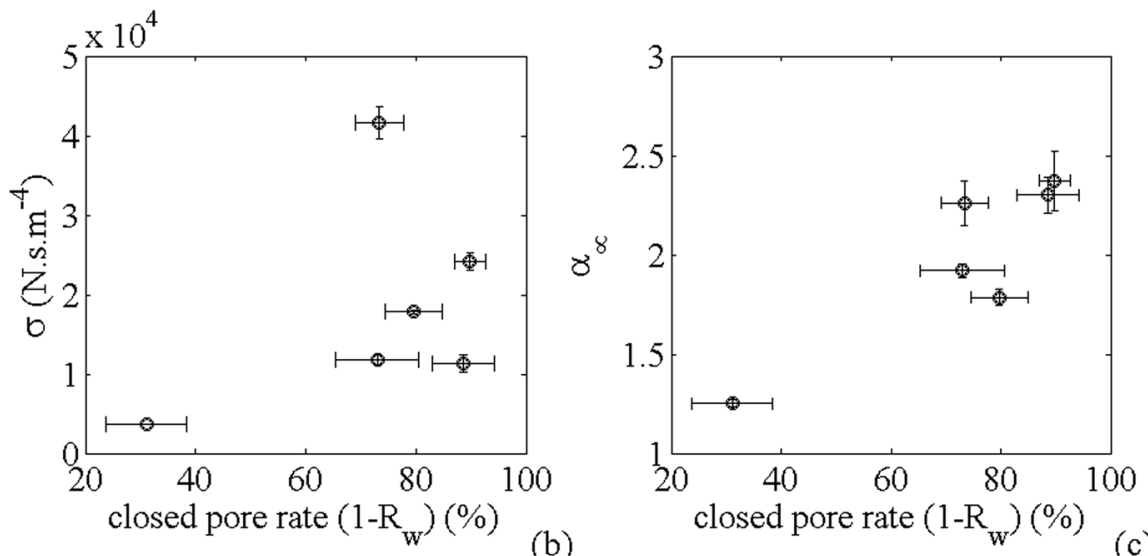

(b)
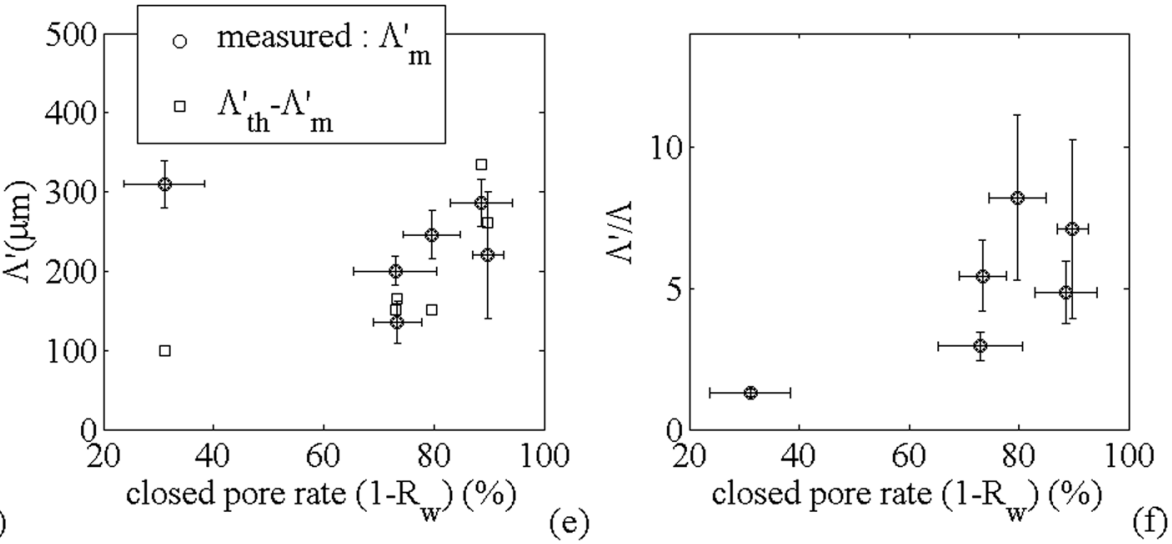

FIG. 6. Nonacoustic properties in terms of closed pore content for the 6 partially reticulated foams.

size. As shown in Figs. 7(b) and 7(d), the materials with the smallest cells still show the smaller characteristic lengths, not the materials with the highest closed pore content. Thus, for these partially reticulated materials, relations between cell size and thermal length, and window size and viscous length are not direct because the reticulation still plays an important role. However, it is found that the $\Lambda^{\prime} / \Lambda$ ratio increases with the closed pore content as shown in Fig. 6(f).


(c)
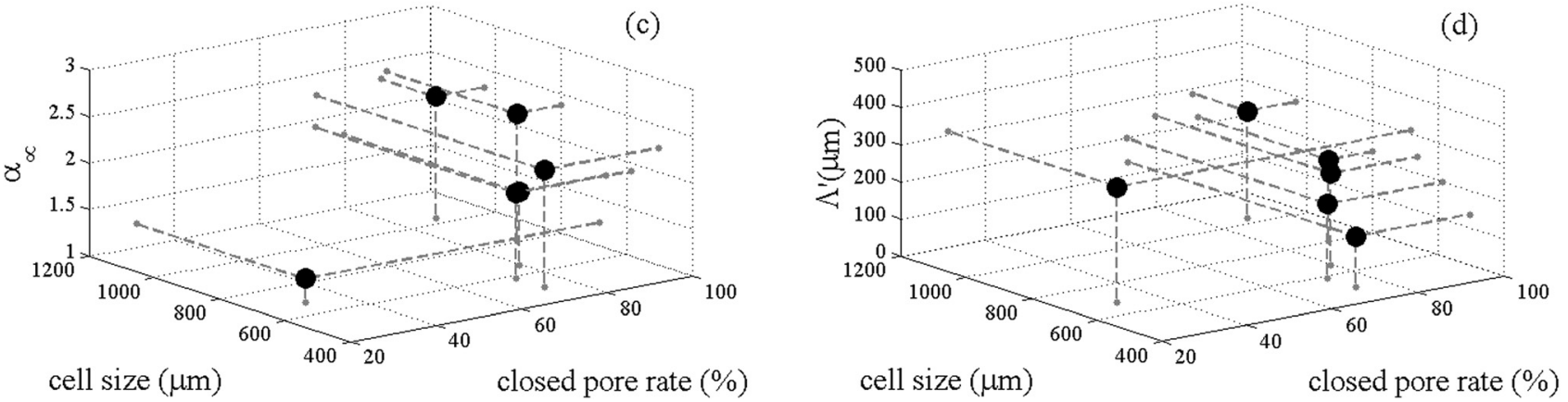

FIG. 7. Nonacoustic properties in terms of closed pore rate and cell size for the 6 partially reticulated foams. 
This ratio goes from $n=1.29 \pm 0.13$ for low closed pore content to $n=8.20 \pm 2.91$ for high closed pore content (i.e., $1-R_{w}=80 \%$ ). This trend is coherent with the observations given by Perrot et al. ${ }^{13}$ from the numerical study of a $2 \mathrm{D}$ hexagonal lattice of solid fibers in air. Indeed, according to Table I of Ref. 13, for a given fiber radius, decreasing the throat size $w$ (i.e., the distance between two consecutive fibers and thus equivalent to the pore size): (i) reduces the cell size, (ii) increases the tortuosity and airflow resistivity, and (iii) increases the ratio $n=\Lambda^{\prime} / \Lambda$. This ratio is equal to 1.71 for a throat size of $w=210 \mu \mathrm{m}$, and reaches 4.45 for $w=20 \mu \mathrm{m}$. The fact that the $\Lambda^{\prime} / \Lambda$ ratio increases with the closed pore content indicates that the viscous phenomena in the pore constrictions created by the presence of membranes are predominant compared to added thermal effects, especially as the number of constrictions increases, the constriction size may decrease and the particle velocity in these constrictions increases.

Note that all of the polyurethane foams (fully and partially reticulated) have similar frame density $\rho_{l}$ with a mean value of $\rho_{1}=25.7 \pm 0.9 \mathrm{~kg} \mathrm{~m}^{-3}$. Furthermore, it is observed that this property does not vary with cell size which is coherent with porosity and cell shape constancy.

Because the presence of membranes closing the faces has an important impact on both acoustic and nonacoustic properties of the foams, the proposed study to link microstructure with nonacoustic properties is now split in two parts for fully and partially reticulated materials: existing scaling laws are first applied to fully reticulated materials and then, are generalized empirically by the use of the reticulation rate $R_{w}$.

\section{LINK MICROSTRUCTURE AND NONACOUSTIC PROPERTIES}

\section{A. Fully reticulated materials: Application of the existing scaling laws}

\section{Porosity $\Phi$, thermal length $\Lambda^{\prime}$}

According to their definition, both porosity and thermal length are purely geometrical macroscopic parameters and thus, can be estimated from geometrical properties of the representative tetrakaidecahedra unit-cell characterized in Sec. II A.

In the case of the porosity, defined as the ratio of the fluid volume $V_{f}$ to the total volume $V_{t}$, Gibson and Ashby ${ }^{7}$ proposed a simple expression for highly porous material with strut material significantly heavier than the interstitial fluid as

$$
\Phi=1-\frac{\rho_{1}}{\rho_{s}}=1-\rho_{r},
$$

TABLE I. Characteristic parameters for tetrakaidecahedra unit-cell with different strut cross-section shape.

\begin{tabular}{lccc}
\hline \hline Strut cross-section shape & & \multicolumn{1}{c}{} \\
\hline$C^{\rho}$ & $3 \pi / 8 \sqrt{2}$ & $\sqrt{3} / 2 \sqrt{2}$ & $(2 \sqrt{3}-\pi) / \sqrt{2}$ \\
$D_{1}$ & $4 \sqrt{2} / 3 \pi$ & $2 \sqrt{2} / 3 \sqrt{3}$ & $2 \sqrt{6} / 3 \pi$ \\
$D_{2}$ & $1 / 2$ & $1 / 3$ & $(12-2 \pi \sqrt{3}) / 3 \pi$ \\
\hline \hline
\end{tabular}

with $\rho_{1}$ the bulk density of the foam, $\rho_{s}$ the density of the strut material, and $\rho_{r}$ the relative density which can be expressed in terms of strut thickness $t$ and strut length $l$ forming the cells as $\rho_{r}=C^{\rho}(t / l){ }^{2} C^{\rho}$ is a constant which depends on the microscopic mechanical properties of the foam, such as cell shape, strut cross section, and joint region shape. ${ }^{7,8}$ In the case of foams having tetrakaidecahedra unit-cell, such as the polyurethane foams under investigation, Perrot et al. ${ }^{8}$ give an analytical expression of the constant $C^{\rho}$ for different strut cross-section shape. Table I remind this constant for three different strut cross-section shapes: circular crosssection with diameter $d(t=d)$; equilateral triangular cross section with edge $a$; concave equilateral triangular crosssection with edge $a$ and a concavity radius $R(R=a)$. Figure 8 (a) compares the porosity predictions derived with Eq. (1) associated to the three strut cross-section shapes with measurements for the nine fully reticulated materials. Note that in this figure, the materials are ordered by increasing value of the airflow resistivity [see Fig. 8(e)]. Porosity results proposed in Fig. 8(a) confirm the results of Perrot et al.: ${ }^{13}$ the porosity increases with the cross section area of the strut. Here, the concave triangular cross section shape, which is closer to the real strut shape observed in SEM pictures, gives the most reliable predictions compared to experimental data (with $\Phi \approx 98 \%$ ).

In the case of the thermal length $\Lambda^{\prime}$, defined as twice the average ratio of the cell volume $V_{f}$ to their wet surface $A$, Perrot et al. $^{8}$ proposed a simple expression using the same tetrakaidecahedra cell shape assumption in the form

$$
\Lambda^{\prime}=D_{1} \frac{l^{2}}{t}-D_{2} t
$$

where $D_{1}$ and $D_{2}$ are numerical constants near unity which can be determined analytically for simple cases. Parameters $D_{1}$ and $D_{2}$ associated to a tetrakaidecahedra unit-cell with different strut cross-section shapes are given in Table I. Figure 8(b) shows that, for the nine fully reticulated foams, the Eq. (2) slightly overestimates (i.e., by a factor between 1.1 and 1.6) the thermal characteristic length but still gives the good order of magnitude. Here, the three cross-section shapes give similar results because the wet area of the struts is barely the same.

\section{Viscous length $\mathrm{\Lambda}$}

In the case of fibrous materials, the viscous length can be predicted analytically from the calculation of the velocity field around a strut with circular cross-section shape, considering that the velocity far from the strut is perpendicular to the strut. In this case, Allard and Atalla ${ }^{1}$ linked the viscous characteristic length $\Lambda$ to the diameter $t$ of a fiber as

$$
\Lambda=\frac{t}{4 \rho_{r}} .
$$

As shown in Fig. 8(c) (with white circles), this simple analytical model gives a good prediction of the viscous length for fully reticulated foams. Thus, fully reticulated polyurethane foams can be considered having a fibrous-like 




(a)

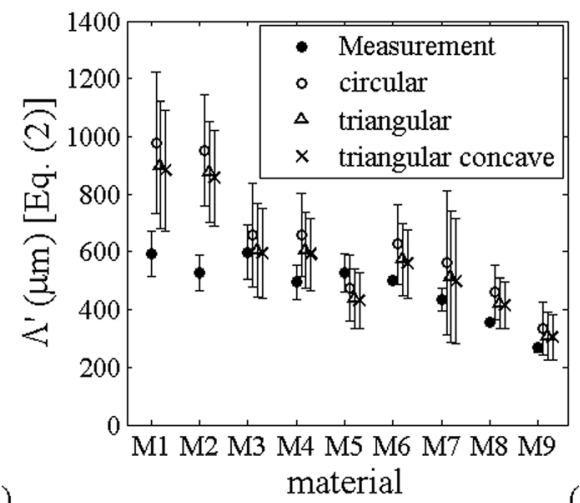

(b)

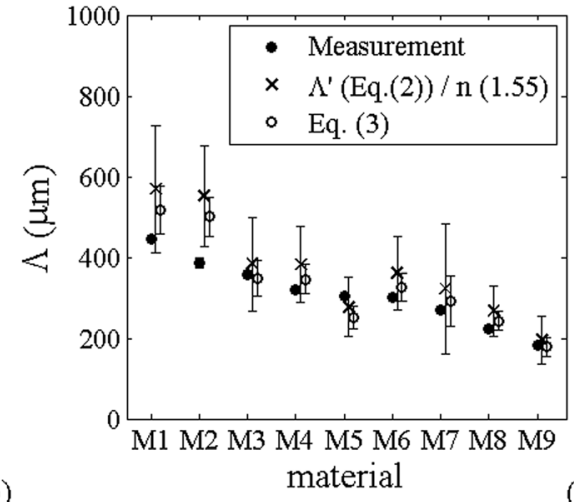

(c)

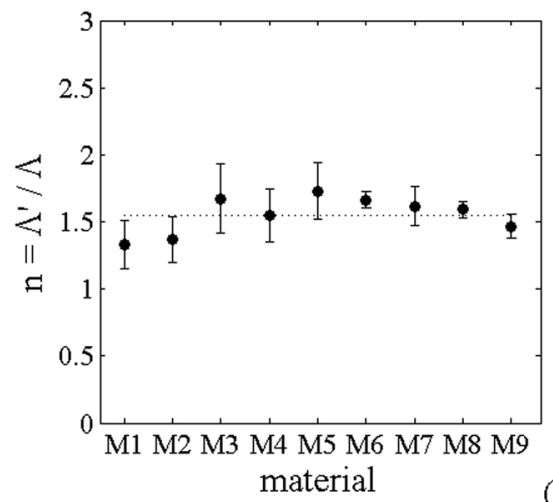

(d)

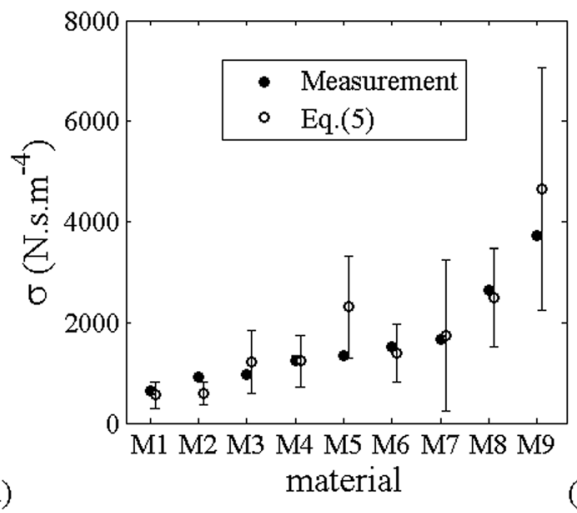

(e)

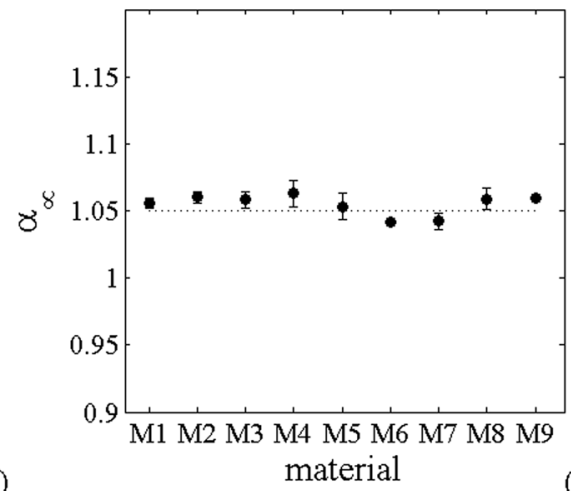

(f)

FIG. 8. Fully reticulated materials: measured nonacoustic properties and estimation from existing scaling laws; expanded uncertainty bars are also shown.

behavior. Using the same assumptions, Allard and Atalla ${ }^{1}$ show that the thermal and viscous characteristic lengths of fibrous materials are linked by a factor 2 as $n=\Lambda^{\prime} / \Lambda=2$ (when the macroscopic air velocity is perpendicular to the direction of the fibers). As mentioned previously and shown in Fig. $8(\mathrm{~d})$, the ratio between the thermal and the viscous characteristic lengths $\left(n=\Lambda^{\prime} / \Lambda\right)$ is close to 1.55 for fully reticulated materials. Thus, the viscous length can be derived from the geometrical estimation of the thermal length of Eq. (2) divided by $n=1.55$. This approximation also gives a good estimation of the viscous length as shown in Fig. 8(c) (see crosses, calculated here for struts having triangular concave cross-section shape).

Note that the viscous characteristic length $\Lambda$ may also be derived from the analytical study of the sound propagation in a pore channel ${ }^{17}$ and given by

$$
\Lambda=\frac{1}{c_{g}} \sqrt{\frac{8 \alpha_{\infty} \eta}{\Phi \sigma}}
$$

where $c_{g}$ is a pore shape dependent constant (it is equal to one for cylindrical geometry), $\eta$ is the viscosity of air and $\sigma$ is the static airflow resistivity. It is not used here since it requires the knowledge of the other intercorrelated nonacoustic parameters.

\section{Airflow resistivity $\sigma$ and tortuosity $\alpha_{\infty}$}

By combining Eqs. (1), (3), and (4), Lind-Nordgren and Göransson $^{9,10}$ proposed a scaling law applied to the airflow resistivity $\sigma$ of porous materials (fibrous and foams) having a porosity and tortuosity close to 1 such as

$$
\sigma=\frac{C^{\sigma}}{t^{2}} \rho_{r}^{2}=C^{\sigma}\left(C^{\rho} \frac{t}{l^{2}}\right)^{2}
$$

In the case of porous materials made of fibers with circular cross-section shape, $C^{\sigma}=128 \alpha_{\infty} \eta / c_{g}{ }^{2}$. It is worth mentioning that this scaling law has a large number of implicit assumptions: propagation in a cylindrical pore $\left[c_{g}=1\right.$ and validity of Eq. (4)], material with low tortuosity $\left(\alpha_{\infty} \approx 1\right)$ and high porosity $(\Phi \approx 100 \%)$ and wave propagation perpendicular to ligament with a circular cross-section shape [validity of Eq. (3)]. Still, it is shown in Fig. 8(e) that it gives surprisingly a satisfactory prediction of the airflow resistivity for the nine fully reticulated polyurethane foams. However, its associated standard deviation seems to increase while cell size decreases (i.e., increasing airflow resistivity), making Eq. (5) more adapted to large cell materials.

Because no direct links between tortuosity and microstructure geometry of fully reticulated polyurethane foams have been observed [see Fig. 5(c)], the tortuosity of these materials is considered equal to the mean value $\alpha_{\infty}=1.05$ [see Fig. 8(f)].

\section{B. Partially reticulated materials: Improvement of the scaling laws}

The main objective of this section is to improve the existing scaling laws to account for the reticulation rate and thus be able predict the nonacoustic properties of partially reticulated foams from microstructure measurements. 


\section{Porosity $\Phi$, thermal length $\Lambda^{\prime}$}

The porosity being defined as the ratio of the fluid volume $V_{f}$ to the total volume $V_{t}, \Phi=V_{f} / V_{t}=V_{f} /\left(V_{f}+V_{s}\right)$, the effect of membranes is not taken into account here since the membrane volume can be neglected in the calculation of the frame volume $V_{s}$. Thus, Eq. (1) is also used here for the partially reticulated ones. Figure 9(a) shows that, as expected, the strut with concave triangular cross-section shape gives the more reliable estimation of the porosity. Note that the materials presented in Fig. 9 are ordered by increasing value of the airflow resistivity [see Fig. 9(e), black points].

According to the thermal characteristic length definition, the reticulation rate may modify its value since it increases the wet surface A. Thus, the thermal characteristic length $\Lambda^{\prime}$ can be rewritten as

$$
\Lambda^{\prime}=\frac{2 V_{f}}{A}=\frac{2 V_{f}}{A_{S}+\left(1-R_{w}\right) A_{w}},
$$

with $A_{s}$ the surface of the struts and $A_{w}$ the total surface of the pores weighted here by the closed pore rate $\left(1-R_{w}\right) . A_{s}$ can be calculated from the perimeter of the strut $P_{s}$ for a given cross-section shape. Assuming a tetrakaidecahedra unit cell, the surface of the 36 struts per cell $A_{s}$, is given by $A_{s}=36 l P_{s} / 3$; the " $1 / 3$ " coefficient accounting for the fact that one strut is shared between three cells. The total surface of the pores is determined from the total surface of the cell $A_{c}$ as $A_{w}=A_{c}-A_{s}$. For a tetrakaidecahedra unit cell, this surface is $A_{c}=(6+12 \sqrt{3}) l^{2}$. Note that for fully reticulated materials $\left(R_{w}=100 \%\right)$, the wet surface area is only the sur- face of the struts $A_{s}$ and the thermal length of Eq. (6) has the simple form of Eq. (2).

It is shown in Fig. 9(b) that Eq. (6) allows a good estimation of the thermal characteristic length $\Lambda^{\prime}$ of partially reticulated materials (not accounting for the presence of membranes induces an overestimation of the thermal length; see white squares). As for fully reticulated materials, the strut cross-section shape has a marginal influence on the thermal characteristic length estimation.

\section{Viscous length $\Lambda$}

Presence of membranes closing the windows decreases the viscous characteristic length and increases the characteristic length ratio $n\left(n=\Lambda^{\prime} / \Lambda\right)$ [see Figs. 6(d) and 6(f)]. Thus, the simple relation between the two characteristic lengths used in the case of fully reticulated materials, i.e., $n=1.55$, can no longer be used for partially reticulated materials. This is illustrated in Fig. 9(c) where the viscous length estimated as half the thermal length of Eq. (6) is greatly overestimated compared to measurements (see gray circles).

Thus from the 6 partially reticulated materials under investigation, an empirical expression of the ratio $n$ is derived in terms of the closed pore content and given by

$$
n=\frac{\Lambda^{\prime}}{\Lambda}=1.55\left(\frac{1}{R_{w}}\right)^{0.676} .
$$

This empirical expression, where the coefficient 0.676 has been determined from curve fitting, is in good agreement with measurements as shown in Fig. 9(d) and allows a

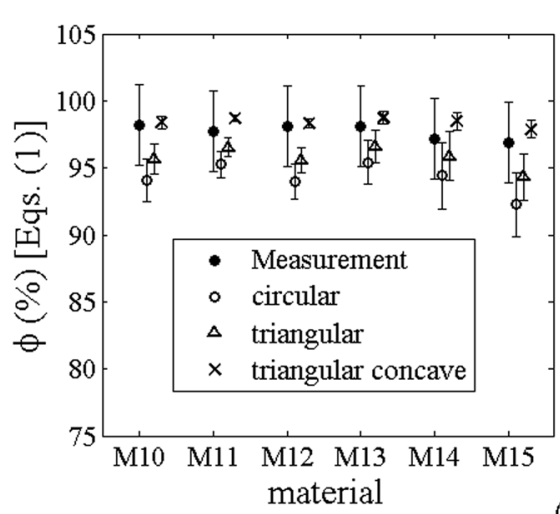

(a)

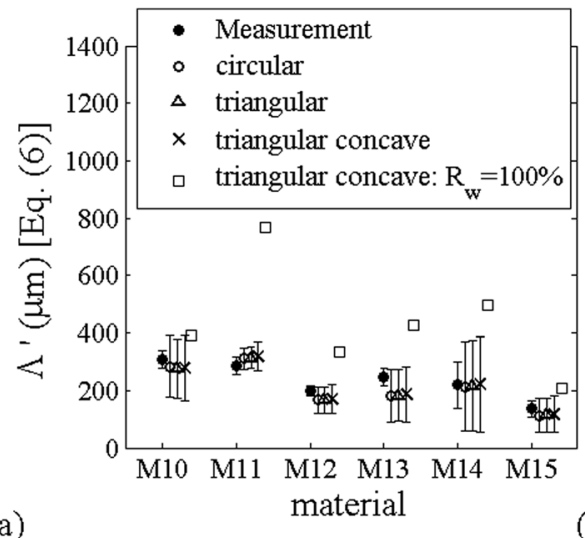

(b)

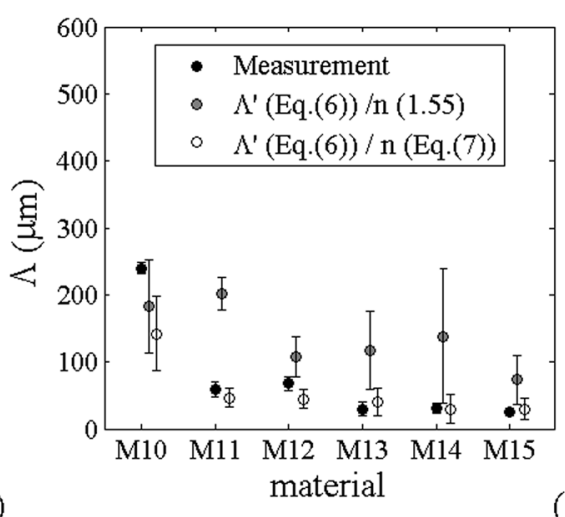

(c)

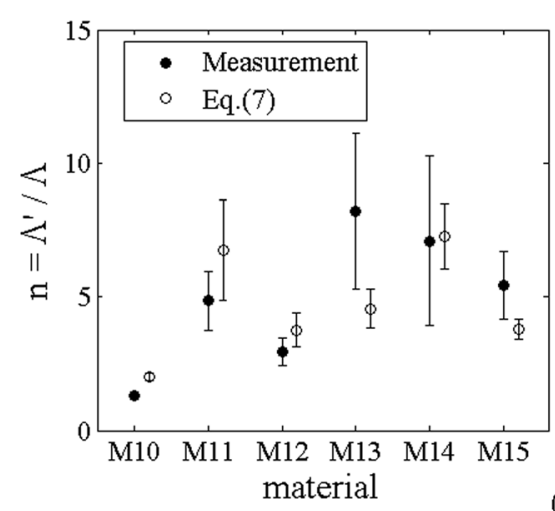

(d)

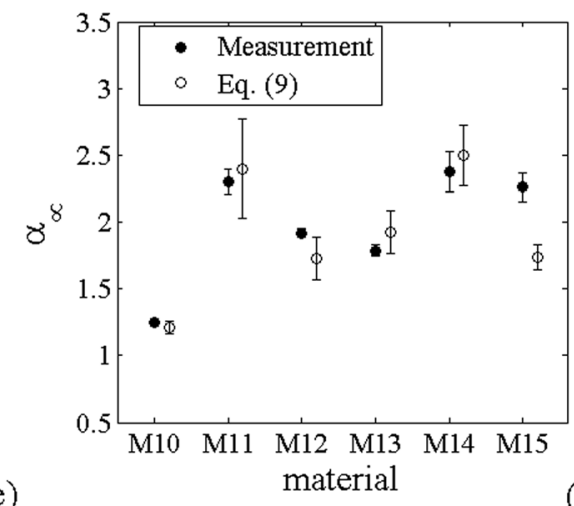

(f)

FIG. 9. Partially reticulated materials: measured nonacoustic properties and estimation from modified scaling laws. Expanded uncertainty bars are also shown. 
satisfactory estimation of the viscous characteristic length [see white circles in Fig. 9(c)].

\section{Airflow resistivity $\sigma$ and tortuosity $\alpha_{\infty}$}

As for the viscous length parameter, the determination of the airflow resistivity of partially reticulated materials using the scaling law developed for fully reticulated materials [Eq. (5)] is no more appropriate [see Fig. 9(e) gray circles] since the presence of membranes greatly affects the permeability of the material. Thus, the following empirical expression is proposed to add the influence of the reticulation rate,

$$
\sigma=C^{\sigma}\left(C^{\rho} \frac{t}{l^{2}}\right)^{2}\left(\frac{1}{R_{w}}\right)^{1.116}
$$

In the same way, because the tortuosity of fully reticulated material is considered equal to the mean value of 1.05, and it has been shown previously this parameter mainly depends on the closed pore content and increases with closed pore content, it is proposed for partially reticulated materials as

$$
\alpha_{\infty}=1.05\left(\frac{1}{R_{w}}\right)^{0.380}
$$

Figures 9(e) and 9(f) show that these two empirical expressions which integrate the reticulation rate in existing scaling law allow a relatively good estimation of the airflow resistivity and tortuosity of partially reticulated materials, still with a large standard deviation associated with the airflow resistivity prediction.

\section{ACOUSTIC PROPERTY}

The acoustic property considered in this study is the normal incidence sound absorption. It is measured in an impedance tube according to standard ISO-10534-2. ${ }^{28}$ Figure 10 presents this coefficient in terms of frequency for 3 materials: the fully reticulated materials M7 and M9 and the partially reticulated material M11. Measurement carried out on a 2 in thick sample and simulation using the "rigid" equivalent fluid model with the nonacoustic parameters linked to microstructure properties from the proposed empirical and

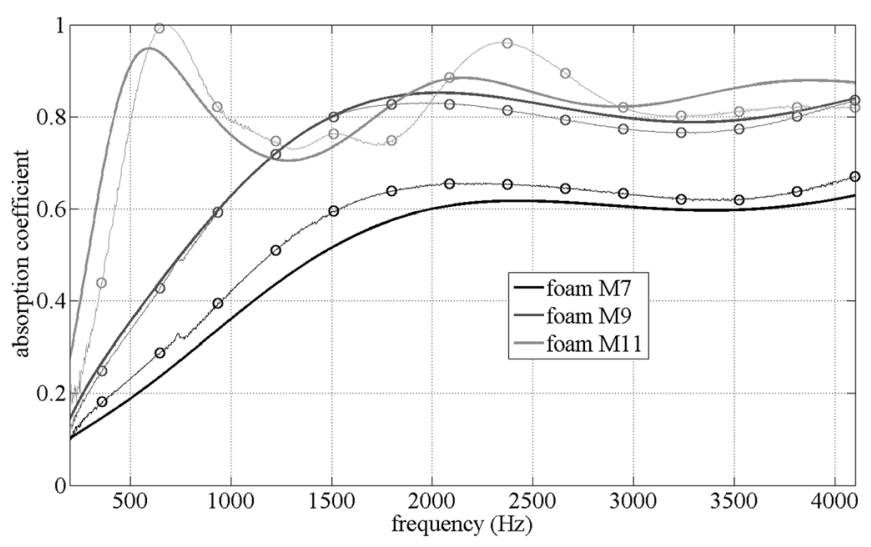

FIG. 10. Absorption coefficient of the fully reticulated materials M7 and M9 and partially reticulated M11: measurements (circle) vs simulations (solid thick line). geometrical expressions [see Eqs. (1), (6)-(9)] are presented. It is shown that simulations carried out using the estimated nonacoustic parameters (see thick solid lines) are in good agreement with measurements (circles). In order to quantify the differences between measurements and simulations for a given material, the Noise Reduction Coefficient (NRC) is computed for the two curves and the relative variation $\triangle \mathrm{NRC}$ in percent is derived as $\triangle \mathrm{NRC}=100$ $\times\left|\mathrm{NRC}_{\text {measurement }}{ }^{-} \mathrm{NRC}_{\text {simulation }}\right| / \mathrm{NRC}_{\text {measurement. }}$. Note that the NRC is the arithmetic average of the sound absorption derived here at the 17 octave bands between 100 and $4000 \mathrm{~Hz}$. For fully reticulated materials M7 and M9, $\Delta$ NRC are $6.8 \%$ and $5.5 \%$, respectively. For all fully reticulated materials, $\triangle \mathrm{NRC}$ is ranging between $0.9 \%$ and $24.7 \%$ with the average value at $10.8 \%$. For partially reticulated material $\mathrm{M} 11, \Delta \mathrm{NRC}=7.8 \%$ and for all partially reticulated materials, $\triangle \mathrm{NRC}$ is ranging between $1.7 \%$ and $12.9 \%$ with the average value at $6.3 \%$. Thus, the estimation of the nonacoustic properties from microstructure analysis and use of Eqs. (1), (6)-(9) is considered satisfactory.

According to Fig. 10, the fully reticulated material M7 with an airflow resistivity of $1677 \mathrm{~N} \mathrm{~s} \mathrm{~m}^{-4}$ shows lower absorption efficiency compared to foam M9 with an airflow resistivity of $3996 \mathrm{~N} \mathrm{~s} \mathrm{~m}^{-4}$ and to the partially reticulated material M11 having an airflow resistivity of $11345 \mathrm{~N} \mathrm{~s}$ $\mathrm{m}^{-4}$. Indeed, it is well known that an increase of the airflow resistivity improves the absorption behavior in the whole frequency range but only until an intermediate value. A too resistive porous material will show poor sound absorption behavior since it would be more difficult for the acoustic wave to penetrate into the material. In the case of the partially reticulated material M11, the sound absorption efficiency is also enhanced at low frequencies because the viscous length is small and the tortuosity is high; which is due to the presence of membranes closing the cells.

\section{CONCLUSION}

In this paper, links between the microstructure of fully and partially reticulated polyurethane foams and their acoustic behavior is investigated. First, the microstructure of 15 isotropic polyurethane foams having similar frame density is characterized from SEM pictures and a representative unitcell with tetrakaidecahedra shape is determined for each foam. Then, existing geometrical formulations and scaling laws which relate microstructure properties (cell size, strut length and thickness) with nonacoustic parameters (porosity, airflow resistivity,...) are applied to fully reticulated foams. This analysis confirms that both porosity and thermal characteristic length of the fully reticulated polyurethane foams can be estimated by simply considering the foam microstructure as a packing of tetrakaidecahedra cells with struts having triangular concave cross section shape. Furthermore, it is shown that these fully reticulated foams can be modeled as fibers lying perpendicular to the acoustic wave or as porous materials with cylindrical pores to estimate the airflow resistivity and viscous characteristic length; all assumptions allowing a satisfactory estimation of the acoustic performance. Then, the existing micro-macro links were improved 
empirically to account for the presence of membranes closing the pores. Despite the limited amount of tested foams, the empirical model is in good agreement with sound absorption measurements of partially reticulated materials. Furthermore, it is shown that the reticulation rate is an important parameter which has a large influence on the acoustic behavior, i.e., it may dominate the cell size influence. Decreasing the reticulation rate increases the airflow resistivity, the tortuosity, the characteristic lengths ratio and the coupling between the frame and the saturating fluid especially for low density materials. Thus, the acoustical efficiency at low frequencies is improved but can be worsened in some higher frequency bands. For fully reticulated materials, decreasing the cell size increases the airflow resistivity and decreases the two characteristic lengths, and thus improves the sound absorption efficiency. However, the airflow resistivity should not be too high (i.e., cell size too small) to maintain good absorption behavior. From a practical point of view, the proposed strategy while heavily experimental allows for a drastic reduction of the computational cost compared to using purely computational methods. More importantly, it offers the advantage of being fully compatible with the physics of the manufacturing process, of the shapes and sizes of the structure. It allows also for isolating the predominant dissipation phenomena occurring at the local scale, and deducing from this knowledge, pertinent modifications of the microstructure (optimization).

\section{ACKNOWLEDGMENTS}

The authors would like to thank the National Sciences and Engineering Research Council of Canada (NSERC) for providing financial support. S. Ghaffari Mosanenzadeh from the University of Toronto is gratefully acknowledged for providing the SEM pictures used in this work.
${ }^{1}$ J. F. Allard and N. Atalla, Propagation of Sound in Porous Media: Modeling Sound Absorbing Materials, 2nd ed. (Wiley, New York, 2009).

${ }^{2}$ D. A. Bies and C. H. Hansen, Appl. Acoust. 13, 357 (1980).

${ }^{3}$ M. Garai and F. Pompoli, Appl. Acoust. 66, 1383 (2005).

${ }^{4}$ N. Kino and T. Ueno, Appl. Acoust. 68, 1439 (2007).

${ }^{5}$ N. Kino and T. Ueno, Appl. Acoust. 69, 575 (2008).

${ }^{6}$ N. Kino and T. Ueno, Appl. Acoust. 69, 325 (2008).

${ }^{7}$ L. J. Gibson and M. F. Ashby, Cellular Solids-Structure and Properties, 2nd ed. (Cambridge University Press, Cambridge, 1997, first published by Pergamon, 1988).

${ }^{8}$ C. Perrot, R. Panneton, and X. Olny, J. Appl. Phys. 101, 113538 (2007).

${ }^{9}$ P. Göransson, Philos. Trans. R. Soc.London, Ser. A 364, 89 (2006).

${ }^{10}$ E. Lind-Nordgren and P. Göransson, J. SoundVib. 329, 753 (2010).

${ }^{11}$ C. Perrot, R. Panneton, and X. Olny, J. Appl. Phys. 102, 074917 (2007).

${ }^{12}$ C. Perrot, F. Chevillotte, and R. Panneton, J. Appl. Phys. 103, 024909 (2008).

${ }^{13}$ C. Perrot, F. Chevillotte, and R. Panneton, J. Acoust. Soc. Am. 124(2), 940 (2008).

${ }^{14}$ L. Boeckx, M. Brennan, K. Verniers, and J. Vandenbroeck, Acta Acust. Acust. 96, 239 (2010).

15 Joint Committee for Guides in Metrology, Evaluation of Measurement Data-Guide to the Expression of Uncertainty in Measurement (JCGM 100:2008, Bur. Intl. Poids et Mesures, Sèvres, 2008).

${ }^{16}$ D. Lafarge, P. Lemarinier, J.-F. Allard, and V. Tarnow, J. Acoust. Soc. Am. 102, 1995 (1997).

${ }^{17}$ D. L. Johnson, J. Koplik, and R. Dashen, J. Fluid Mech. 176, 379 (1987).

${ }^{18}$ Y. Salissou and R. Panneton, J. Appl. Phys. 101, 124913.1 (2007).

${ }^{19}$ M. R. Stinson and G. A. Daigle, J. Acoust. Soc. Am. 83, 2422 (1988).

${ }^{20}$ M. Melon and B. Castagnede, J. Acoust. Soc. Am. 98, 1228 (1995).

${ }^{21}$ Z. E. A. Fellah, S. Berger, W. Lauriks, C. Depollier, C. Aristégui, and J.-Y. Chapelon, J. Acoust. Soc. Am. 113(5), 2424 (2003).

${ }^{22}$ R. Panneton and X. Olny, J. Acoust. Soc. Am. 119(4), 2027 (2006).

${ }^{23}$ X. Olny and R. Panneton, J. Acoust. Soc. Am. 123(2), 814 (2008).

${ }^{24}$ Y. Salissou and R. Panneton, J. Acoust. Soc. Am. 128(5), 2868 (2010).

${ }^{25}$ O. Doutres, Y. Salissou, N. Atalla, and R. Panneton, Appl. Acoust. 71(6), 506 (2010).

${ }^{26}$ S. R. Pride, F. D. Morgan, and A. F. Gangi, Phys. Rev. B 47(9), 4964 (1993).

${ }^{27}$ D. Lafarge, (in French), Ph.D. Thesis, Université du Maine (1993).

${ }^{28}$ Anonymous, "Acoustics - Determination of sound absorption coefficient and impedance in impedance tubes. Part 2: Transfer-function method," International Standard ISO-10534-2 (1998). 
Journal of Applied Physics is copyrighted by the American Institute of Physics (AIP). Redistribution of journal material is subject to the AIP online journal license and/or AIP copyright. For more information, see http://ojps.aip.org/japo/japcr/jsp 